\title{
Primary thyroid leiomyosarcoma - case report and literature review
}

\author{
Cherry $\mathrm{TJ}^{1 *}$, Tasevski $\mathrm{R}^{1,2}$ and $\mathrm{Li}^{\mathbf{1}}$ \\ ${ }^{1}$ Department of General Surgery, The Royal Melbourne Hospital, Parkville, Victoria, Australia \\ ${ }^{2}$ Department of Surgery, University of Melbourne, Parkville, VIC, Australia
}

\begin{abstract}
Primary thyroid leiomyosarcoma is a rare malignancy. Patients usually present with advanced disease and less than $10 \%$ survive beyond one year. In making the diagnosis it is difficult to distinguish cytologically between spindle cell malignancies. We present a 66-year old male patient who required open incisional biopsy to obtain the diagnosis of thyroid leiomyosarcoma and allow for appropriate management.
\end{abstract}

\section{Case History}

A 66-year old man presented to the Emergency Department with a large, palpable, multi-nodular goitre and a six-month history of voice changes and intermittent stridor. He was clinically and biochemically euthyroid. He had a past medical history of congenital arachnoid cyst, gout, asthma and was taking Warfarin for a previous deep vein thrombosis. He had a computed tomography (CT) scan of his neck which demonstrated a diffusely enlarged thyroid measuring $8 \times 6 \times$ $11 \mathrm{~cm}$ with retrosternal extension and internal calcification (Figure 1). The inferior aspect abutted the left braciocephalic vein which was thrombosed. A small nodule also protruded into the trachea, suspicious for direct invasion. He had asymmetry of the vocal cords, suggesting left recurrent laryngeal nerve palsy, as well as enlarged pretracheal lymph nodes and two nodules within the lungs concerning for metastases. The thyroid appearance was consistent with invasive malignancy.

An ultrasound of the thyroid revealed a $2 \mathrm{~cm}$ hypoechoic nodule in the left lobe, and multiple heterogenous nodules with rim calcification and cystic changes in the right lobe (Figure 2). A fine needle aspiration (FNA) was performed on the largest heterogenous nodule. The cytological description was that of scant atypical spindle cells showing elongated nuclei, granular chromatin and wispy cytoplasm. This was reported as atypia of undetermined significance (Bethesda 3) [1]. Due to the suspicion of a high-grade
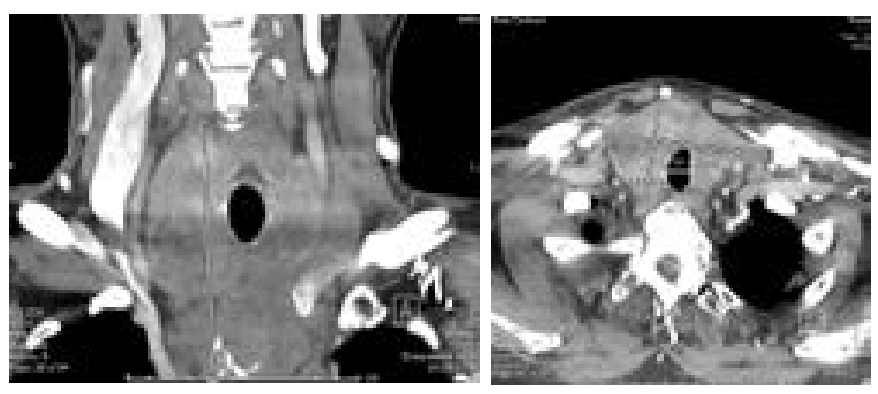

Figure 1. Axial and coronal slices of an intravenous contrast-enhanced CT of the neck demonstrating a large thyroid mass

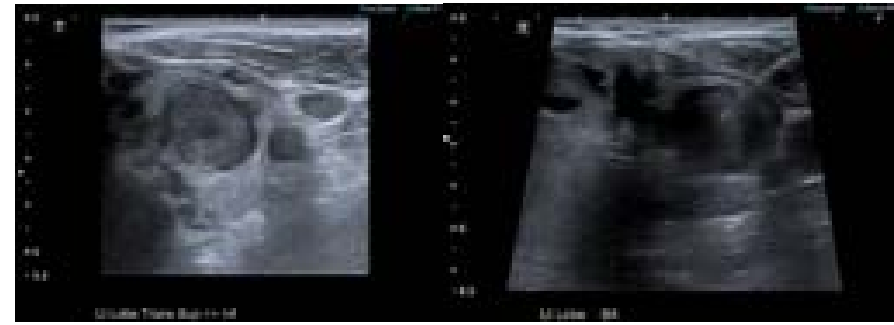

Figure 2. Ultrasound images of the left lobe of the thyroid. Left image: A large heterogenous lesion within the left lobe of the thyroid. Right image: Core biopsy being performed of this lesion

malignancy, the patient proceeded to have core biopsy samples taken under ultrasound guidance with the aim of obtaining a tissue diagnosis. These demonstrated spindle stromal cells without any normal thyroid parenchyma. CKAE1/3 and TTF1 immunostains were negative and this was unfortunately insufficient to provide a histological diagnosis.

After discussion at the Thyroid Cancer Multidisciplinary Meeting, an incisional biopsy was deemed necessary to obtain a definitive diagnosis. The patient was consented for an open diagnostic incisional biopsy with the option to proceed to total thyroidectomy, central neck dissection +/- sternotomy if feasible. The left lobe was approached as for lobectomy, with a suspicious nodule directly visualised. Dissection was very difficult, with no native tissue planes remaining between the thyroid gland and adjacent structures. The left lobe was very stiff and extended retrosternally; a sternotomy would have been required for complete lobectomy. A frozen section sample was sent intra-operatively and demonstrated features suggestive of spindle cell tumour. Due to the significant operative difficulty of proceeding with excisional surgery,

${ }^{*}$ Correspondence to: Tiffany Cherry, Department of General Surgery, Royal Melbourne Hospital, Grattan St, Parkville, VIC, Australia, E-mail: tiffany. cherry@mh.org.au

Key words: thyroid cancer, leiomyosarcoma

Received: April 02, 2019; Accepted: April 11, 2019; Published: April 15, 2019 
further samples were taken for diagnostic purposes and resection was not performed.

The tissue obtained from the open, incisional biopsy demonstrated a malignant spindle cell tumour, with features favouring leiomyosarcoma. Immunohistochemistry was positive for smooth muscle actin and myosin heavy chain but negative for S100, AE1/3, CAM5.2, TTF1, thyroglobulin, PAX8, EMA, CD34, CD31, SOX10, Desmin and Calcitonin. A staging positron emission tomography (PET) scan was also performed demonstrating locally invasive thyroid malignancy extending into the trachea with multiple pulmonary metastases (Figure 3). After multi-disciplinary oncology discussion, he received local radiation therapy. He elected not to proceed with systemic chemotherapy and received palliative care in the community before passing away approximately four months after diagnosis.

\section{Discussion}

Leiomyosarcoma (LMS) is a tumour demonstrating differentiation towards smooth muscle and accounts for less than $0.02 \%$ of primary thyroid malignancies. It is postulated to develop from the smooth muscle within the veins of the thyroid [2]. There have only been 29 case reports of primary thyroid leiomyosarcoma in English medical literature. Two recent literature reviews provide concise summaries of these reports $[3,4]$.

The primary presenting complaint for almost all reported adult cases is the rapid growth of a neck mass which may be associated with dysphagia, odynophagia or dysphonia. Obtaining an accurate diagnosis can be difficult, and there are no imaging features on ultrasound, $\mathrm{CT}$, magnetic resonance imaging (MRI) or PET scanning that definitively differentiate LMS from other invasive thyroid malignancies $[3,4]$. It is also difficult to distinguish between other spindle-cell malignancies on tissue samples, particularly fine needle aspirates. Immunohistochemically, LMS tumour cells are positive for vimentin and SMA while variably positive for desmin, HHF35 and H-caldesmon $[2,5,6]$.

Due to its rarity, no consensus guidelines exist to determine appropriate management. Surgical approaches described include total thyroidectomy with extended neck dissection through to debulking for symptom reduction. There is no survival or significant morbidity benefit demonstrated with any surgical technique. No chemotherapy, radiotherapy or monoclonal antibody treatment regimen has

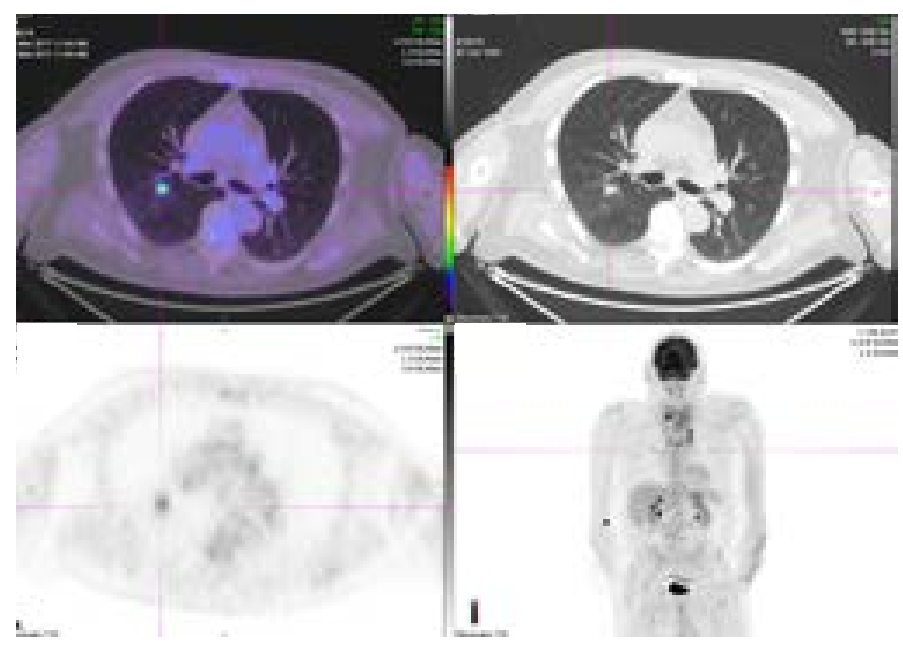

Figure 3. PET-avid nodule within the right lung demonstrated a survival benefit $[4,7,8]$. The prognosis is poor, with less than $10 \%$ of patients surviving beyond a year $[4,9]$.

The case presented reinforces some of the patterns previously demonstrated in reports of this rare disease. Our patient presented with a rapidly growing neck mass and stridor. There were metastases at the time of presentation and a tissue diagnosis was difficult to obtain via the usual diagnostic pathway. The open incisional biopsy was a crucially important step in his treatment algorithm, as it provided a definitive diagnosis that allowed for appropriate palliative management without subjecting him to the morbidity of futile surgery. No curative treatment options were available to him and he died four months after his initial presentation. LMS should be considered in the differential diagnosis of the patient presenting with a rapidly growing thyroid malignancy and cases should continue to be reported to allow for the identification of pathophysiological mechanisms and diagnostic and treatment algorithms.

\section{References}

1. Haugen BR, Alexander EK, Bible KC, Doherty GM, Mandel SJ, et al. (2016) 2015 American Thyroid Association Management Guidelines for Adult Patients with Thyroid Nodules and Differentiated Thyroid Cancer: The American Thyroid Association Guidelines Task Force on Thyroid Nodules and Differentiated Thyroid Cancer. Thyroid 26: 1-133. [Crossref]

2. Thompson LD, Wenig BM, Adair CF, Shmookler BM, Heffess CS (1997) Primary smooth muscle tumors of the thyroid gland. Cancer 79: 579-587. [Crossref]

3. Sahin MI, Vural A, Yuce I, Cagli S, Deniz K, et al. (2016) Thyroid leiomyosarcoma: presentation of two cases and review of the literature. Brazili J otorhinolaryngolo 82: 715-21.

4. Vujosevic S, Krnjevic D, Bogojevic M, Vuckovic L, Filipovic A, et al. (2019) Primary leiomyosarcoma of the thyroid gland with prior malignancy and radiotherapy: A case report and review of literature. World J Clin Cases 7: 473-81. [Crossref]

5. Kawahara E, Nakanishi I, Terahata S, Ikegaki S (1988) Leiomyosarcoma of the thyroid gland. A case report with a comparative study of five cases of anaplastic carcinoma. Cancer 62: 2558-2563. [Crossref]

6. Kaur A, Jayaram G (1990) Thyroid tumors: cytomorphology of medullary, clinically anaplastic, and miscellaneous thyroid neoplasms. Diagn Cytopathol 6: 383-9. [Crossref]

7. Wang TS, Ocal IT, Oxley K, Sosa JA (2008) Primary leiomyosarcoma of the thyroid gland. Thyroid 18: 425-428. [Crossref]

8. Akcam T, Oysul K, Birkent H, Gerek M, Yetiser S (2005) Leiomyosarcoma of the head and neck: report of two cases and review of the literature. Auris Nasus Larynx 32: 209-212. [Crossref]

9. Mansouri H, Gaye M, Errihani H, Kettani F, Gueddari BE (2008) Leiomyosarcoma of the thyroid gland. Acta Otolaryngol 128: 335-336. [Crossref]

Copyright: (C2019 Cherry TJ. This is an open-access article distributed under the terms of the Creative Commons Attribution License, which permits unrestricted use, distribution, and reproduction in any medium, provided the original author and source are credited. 